\title{
Microsatellite loci for Urochloa decumbens (Stapf) R.D. Webster and cross-amplification in other Urochloa species
}

\author{
Rebecca C. U. Ferreira' ${ }^{1}$, Letícia J. Cançado ${ }^{2}$, Cacilda B. do Valle ${ }^{3}$, Lucimara Chiari ${ }^{3}$ and Anete P. de Souza ${ }^{1,4^{*}}$
}

\begin{abstract}
Background: Forage grasses of the African genus Urochloa (syn. Brachiaria) are the basis of Brazilian beef production, and there is a strong demand for high quality, productive and adapted forage plants. Among the approximately 100 species of the genus Urochloa, Urochloa decumbens is one of the most important tropical forage grasses used for pastures due to several of its agronomic attributes. However, the level of understanding of these attributes and the tools with which to control them at the genetic level are limited, mainly due to the apomixis and ploidy level of this species. In this context, the present study aimed to identify and characterize molecular microsatellite markers of $U$. decumbens and to evaluate their cross-amplification in other Urochloa species.
\end{abstract}

Findings: Microsatellite loci were isolated from a previously constructed enriched library from one $U$. decumbens genotype. Specific primers were designed for one hundred thirteen loci, and ninety-three primer pairs successfully amplified microsatellite regions, yielding an average of 4.93 alleles per locus. The polymorphism information content (PIC) values of these loci ranged from 0.26 to 0.85 (average 0.68), and the associated discriminating power (DP) values ranged from 0.22 to 0.97 (average 0.77 ). Cross-amplification studies demonstrated the potential transferability of these microsatellites to four other Urochloa species. Structure analysis revealed the existence of three distinct groups, providing evidence in the allelic pool that $U$. decumbens is closely related to Urochloa ruziziensis and Urochloa brizantha. The genetic distance values determined using Jaccard's coefficient ranged from 0.06 to 0.76 .

Conclusions: The microsatellite markers identified in this study are the first set of molecular markers for $U$. decumbens species. Their availability will facilitate understanding the genetics of this and other Urochloa species and breeding them, and will be useful for germplasm characterization, linkage mapping and marker-assisted selection.

Keywords: Enriched library, Forage, Signalgrass, Simple sequence repeat, Transferability

\section{Background}

It has been estimated that 167 million hectares of pasture land in Brazil is used to feed a herd of approximately 208 million head of cattle [1]. These pastures consist mainly of forage grasses of the genus Urochloa (syn. Brachiaria), which were introduced from Africa [2]. These forage

\footnotetext{
*Correspondence: anete@unicamp.br

${ }^{4}$ Departamento de Biologia Vegetal, Instituto de Biologia, Universidade Estadual de Campinas (UNICAMP), Cidade Universitária Zeferino Vaz, CP 6109, Campinas, SP CEP 13083-862, Brazil

Full list of author information is available at the end of the article
}

grasses have greatly contributed to the development of the national cattle industry of Brazil, establishing Brazil as the second largest beef producer and the main beef exporter in the world. The competitive advantage of cattle production in Brazil is the exclusive use of pasture [3]. Moreover, Brazil is the largest producer and exporter of tropical forage seeds in the world [2].

One of the most widely cultivated species of Urochloa is Urochloa decumbens Stapf., particularly $U$. decumbens cv. 'Basilisk'. This species exhibits exceptional adaptation to the poor and acidic soils that are typical of the tropics 
and lead to good animal performance [4]. However, the molecular genetic information regarding this species is limited, mainly due to its reproducing predominantly via apomixis and because its ploidy levels range from diploid to pentaploid [5].

The need for new more productive and efficient cultivars has inspired the search for new tools to facilitate the selection process [3]. Thus, genetic and genomic studies are essential to advancing breeding programs via a better understanding of the genetic structure of the species. These types of studies can be conducted by using molecular tools, such as molecular markers.

Among all molecular markers, one of the most effective for plant genetics studies is the microsatellite, also known as the SSR (Simple Sequence Repeat). These markers are highly informative due to their multi-allelic nature, codominant inheritance, high transferability and broad distribution in the genomes of the species [6-8].

Whereas some microsatellite markers for Urochloa species have been developed [9-13], specific microsatellite markers for $U$. decumbens have not been reported. Specific microsatellite molecular markers can be very useful in assessing the genetic diversity of germplasms, performing linkage mapping, identifying quantitative trait loci (QTL), performing genome-wide selection and marker-assisted selection, and facilitating molecular based breeding to improve the economically importance characteristics of a species [6,7]. Moreover, microsatellite markers identified in species with little genome information may be used for cross-amplification between related species [14].

The aims of the present study were to identify and characterize the first set of microsatellite markers for $U$. decumbens and to test their transferability to four other Urochloa species ( $U$. brizantha, $U$. dictyoneura, $U$. humidicola and $U$. ruziziensis).

\section{Methods}

Thirty-four Urochloa genotypes were obtained from the Embrapa Beef Cattle collection, in Campo Grande, MS, Brazil for marker validation. Twenty of these genotypes are represented by $U$. decumbens accessions, six genotypes are intra-specific hybrids of the same species and the other eight genotypes are represented by two different germplasm accessions each from $U$. brizantha, $U$. humidicola, $U$. dictyoneura and $U$. ruziziensis. These other Urochloa species were used for the cross-amplification tests. The annotation numbers, accession numbers (as recorded in the Embrapa- BRA-, in the Embrapa Beef Cattle- EBC- and in the Center for Tropical AgricultureCIAT- databases), genotypes, species identified, their mode of reproduction and the origin of the genotypes are shown in Table 1.
Genomic DNA was isolated from fresh leaves using the CTAB method [15]. The purity and concentration of the isolated DNA were determined using a NanoDrop1000 (Thermo) spectrophotometer and by electrophoresis in a $0.8 \%$ agarose gel that was subsequently stained with ethidium bromide $\left(5 \mu \mathrm{g} / \mathrm{mL}^{-1}\right)$.

In a previous study, a microsatellite-enriched library of one $U$. decumbens genotype was constructed using the method described by Billotte et al. [16]. The sequences were then treated as described previously [9]. The microsatellites were identified using MISA software [17], and only mononucleotides with 12 or more repeats, dinucleotides with six or more repeats, trinucleotides with four or more repeats, and tetra, penta, and hexanucleotides with three or more repeats were considered. The DNA sequences determined in this study were deposited in GenBank under the accession numbers shown in Table 2.

After the primer pairs were designed using Primer3Plus software [18], we added a M13 tail ( $5^{\prime} \mathrm{CAC}$ GACGTTGTAAAACGAC-3') to each forward primer. Polymerase chain reaction (PCR) assays were conducted as described previously [9]. The amplified products were separated by electrophoresis through $3 \%$ agarose gels prior to vertical electrophoresis through $6 \%$ denaturing polyacrylamide gels. The gels were then silver stained [19], and the product sizes were determined by comparison to those of a $10 \mathrm{bp}$ DNA ladder (Invitrogen, Carlsbad, CA, USA).

We considered only the strongest bands because the less intense bands might have been stutter bands and an SSR was considered transferable when a band of the expected size was amplified via PCR and an appropriate SSR pattern was observed. Each SSR allele was treated as dominant due to the high ploidy levels of the genotypes; thus, this analysis was based on the presence (1) or absence ( 0 ) of a band in the polyacrylamide gels.

The genetic distance among the genotypes was evaluated according to Jaccard's coefficient [20] based on a binary matrix constructed using the molecular data. This analysis was conducted using the software package NTSYSpc 2.11X [21]. An unrooted tree was constructed using the weighted neighbor-joining method (NJ) using DARwin 6.0.010 software [22].

The set of molecular data was also analyzed using the admixture model of STRUCTURE software version 2.3.4 [23] to infer the population structure of the 34 genotypes. The admixture model was tested using a period of burnin with 100,000 iterations and a run length of 200,000. The number of $\mathrm{K}$ (clusters) was set from 2 to 20 . To infer the appropriate number of clusters in our data, we used the $\Delta \mathrm{K}$ statistic, which represents the rate of change in the $\log$ probability of the data between successive $\mathrm{K}$ 
Table 1 Genotypes of $U$. decumbens and four other species of the genus Urochloa that were used to characterize the microsatellite markers and analyze their levels of transferability

\begin{tabular}{|c|c|c|c|c|c|c|c|}
\hline AN & CIAT & BRA & EBC & Origin & MR & Genotype & Species \\
\hline 1 & 16494 & 004448 & D005 & Kenya & SEX & Germplasm accession & U. decumbens \\
\hline 2 & 16495 & 004456 & D006 & Kenya & SEX & Germplasm accession & U. decumbens \\
\hline 3 & 16497 & 004472 & D007 & Kenya & $\mathrm{APO}$ & Germplasm accession & U. decumbens \\
\hline 4 & 16498 & 004481 & D008 & Kenya & $\mathrm{APO}$ & Germplasm accession & U. decumbens \\
\hline 5 & 16499 & 004499 & D009 & Kenya & $\mathrm{APO}$ & Germplasm accession & U. decumbens \\
\hline 6 & 16500 & 004502 & D010 & Kenya & $\mathrm{APO}$ & Germplasm accession & U. decumbens \\
\hline 7 & 16501 & 004511 & D011 & Kenya & $\mathrm{APO}$ & Germplasm accession & U. decumbens \\
\hline 8 & 16504 & 004545 & D014 & Kenya & $\mathrm{APO}$ & Germplasm accession & U. decumbens \\
\hline 9 & 26295 & 004651 & D024 & Rwanda & SEX & Germplasm accession & U. decumbens \\
\hline 10 & 26300 & 004707 & D028 & Rwanda & $\mathrm{APO}$ & Germplasm accession & U. decumbens \\
\hline 11 & 26304 & 004740 & D032 & Rwanda & $\mathrm{APO}$ & Germplasm accession & U. decumbens \\
\hline 12 & 26308 & 004782 & D035 & Rwanda & SEX & Germplasm accession & U. decumbens \\
\hline 13 & 16491 & 004421 & D036 & Kenya & $\mathrm{APO}$ & Germplasm accession & U. decumbens \\
\hline 14 & 26306 & 004766 & D040 & Rwanda & SEX & Germplasm accession & U. decumbens \\
\hline 15 & 6370 & 000116 & D059 & Unknown & $\mathrm{APO}$ & Germplasm accession & U. decumbens \\
\hline 16 & 16100 & 001961 & D061 & Unknown & $\mathrm{APO}$ & Germplasm accession & U. decumbens \\
\hline 17 & NA & 001996 & D070 & Unknown & $\mathrm{APO}$ & Germplasm accession & U. decumbens \\
\hline 18 & 6298 & 000060 & D077 & Unknown & $\mathrm{APO}$ & Germplasm accession & U. decumbens \\
\hline 19 & - & - & D024/27 & CNPGC & SEX & Tetraploidized accession & U. decumbens \\
\hline 20 & 606 & 001058 & D062 & Uganda & $\mathrm{APO}$ & Germplasm accession & U. decumbens \\
\hline 21 & - & - & $\mathrm{R} 10$ & CNPGC & NA & Hybrid & U. decumbens \\
\hline 22 & - & - & R44 & CNPGC & $\mathrm{APO}$ & Hybrid & U. decumbens \\
\hline 23 & - & - & $\mathrm{R} 125$ & CNPGC & NA & Hybrid & U. decumbens \\
\hline 24 & - & - & R144 & CNPGC & $\mathrm{APO}$ & Hybrid & U. decumbens \\
\hline 25 & - & - & $\mathrm{R} 146$ & CNPGC & NA & Hybrid & U. decumbens \\
\hline 26 & - & - & R182 & CNPGC & NA & Hybrid & U. decumbens \\
\hline 27 & 16186 & 007889 & DT157 & Ethiopia & $\mathrm{APO}$ & Germplasm accession & U. dictyoneura \\
\hline 28 & 16188 & 007901 & DT159 & Ethiopia & $\mathrm{APO}$ & Germplasm accession & U. dictyoneura \\
\hline 29 & NA & $\mathrm{NA}$ & R044 & Unknown & SEX & Germplasm accession & U.ruziziensis \\
\hline 30 & 26163 & 005568 & $\mathrm{R} 102$ & Burundi & SEX & Germplasm accession & U. ruziziensis \\
\hline 31 & 16125 & 002844 & B112 & Ethiopia & $\mathrm{APO}$ & Germplasm accession & U. brizantha \\
\hline 32 & 26110 & 004308 & B178 & Burundi & $\mathrm{APO}$ & Germplasm accession & U. brizantha \\
\hline 33 & 26149 & 005118 & H016 & Burundi & APO & Germplasm accession & U. humidicola \\
\hline 34 & 6369 & 000370 & $\mathrm{H} 126$ & Unknown & APO & Germplasm accession & U. humidicola \\
\hline
\end{tabular}

AN annotation number, CIAT Center for Tropical Agriculture, BRA codes from Embrapa, CNPGC National Center for Research on Beef Cattle, EBC codes from Embrapa Beef Cattle, MR mode of reproduction- apomictic or sexual, NA not available

values rather than the log probability of the data [24]. We retained the $\mathrm{K}$ value corresponding to the highest value of $\Delta \mathrm{K}$ obtained using the online tool Structure Harvester [25].

The polymorphism information content (PIC) values were calculated to evaluate the levels of marker informativeness and to help choose primers for future studies [26]. To compare the efficacies of the markers used for varietal identification, the discrimination power (DP) value was determined for each primer [27].

\section{Results}

We analyzed 281 contigs, of which 128 were found to contain SSR. One hundred fifty-five SSR motifs were found, with the perfect microsatellite being the most abundant. Dinucleotide repeats were the most abundant class of microsatellite detected (59.36\%), followed by tetranucleotide (18.71\%), trinucleotide (12.26\%), mononucleotide $(3.87 \%)$, hexanucleotide $(3.22 \%)$ and pentanucleotide $(2.58 \%)$ repeats. Furthermore, $22 \%$ of the microsatellite motifs were classified as class I motifs 
Table 2 Description of the 93 SSR markers developed for U. decumbens

\begin{tabular}{|c|c|c|c|c|c|c|c|c|}
\hline SSR locus & $\begin{array}{l}\text { GenBank accession } \\
\text { number }\end{array}$ & Primer sequences $\left(5^{\prime}-3^{\prime}\right)$ & Repeat motif & $\mathrm{Ta}\left({ }^{\circ} \mathrm{C}\right)^{\mathrm{a}}$ & Size (bp) & $N A^{b}$ & PIC $^{c}$ & DPd \\
\hline Dec01 & KT587691 & $\begin{array}{l}\text { F_CAAACGACTGCTGATGATGG } \\
\text { R_TGAGAGGCTAAGAG/CAACCTG }\end{array}$ & $(A C)_{16}$ & $65^{\circ}$ & $250-280$ & 5 & 0.68 & 0.89 \\
\hline Dec03 & KT587692 & $\begin{array}{l}\text { F_AACTGAACGCTGCTTGGTCT } \\
\text { R_GGTCCGGAATAAAAAGCACA }\end{array}$ & $(\mathrm{GT})_{6}$ & $65^{\circ}$ & $240-260$ & 3 & 0.58 & 0.63 \\
\hline Dec05 & KT587693 & $\begin{array}{l}\text { F_GGGCTCCTCATCAGCAGTAG } \\
\text { R_GATGCCTCTCGGGACTATCA }\end{array}$ & $(\mathrm{GAC})_{4}$ & $65^{\circ}$ & $132-140$ & 4 & 0.61 & 0.54 \\
\hline Dec06 & KT587694 & $\begin{array}{l}\text { F_GTTCATGGGGGCAATCAGT } \\
\text { R_CGTGATGTCTGAACGGATGA }\end{array}$ & $(\mathrm{CTGG})_{3}$ & $65^{\circ}$ & $120-130$ & 4 & 0.70 & 0.54 \\
\hline Dec07 & KT587695 & $\begin{array}{l}\text { F_CGAACACATTCACATACAACA } \\
\text { R_CTGTCGGATTTATTTGCATTA }\end{array}$ & $(A C)_{7}$ & $65^{\circ}$ & $226-242$ & 5 & 0.74 & 0.87 \\
\hline Dec09 & KT587696 & $\begin{array}{l}\text { F_GCCCAACTGGAATGTGCTA } \\
\text { R_CGACGTCCTTGTTGTTTGTC }\end{array}$ & $(\mathrm{TC})_{9}$ & $65^{\circ}$ & $240-280$ & 5 & 0.72 & 0.91 \\
\hline Dec10 & KT587697 & $\begin{array}{l}\text { F_GACGTCGAGGACAAACAACA } \\
\text { R_TCCTTACCCTTGCGATTCAC }\end{array}$ & $(\mathrm{CAAG})_{3}$ & $65^{\circ}$ & $216-256$ & 6 & 0.79 & 0.86 \\
\hline Dec11 & KT587698 & $\begin{array}{l}\text { F_GGGGGAAAATGAGACAGACA } \\
\text { R_GCTAACCAGACAGCCACCAC }\end{array}$ & $(A G)_{16}$ & $65^{\circ}$ & 154-198 & 8 & 0.80 & 0.94 \\
\hline Dec12 & KT587699 & $\begin{array}{l}\text { F_CTCACACCCTCCTTCTGCTG } \\
\text { R_CGATCGCTCCCTACTAGTGC }\end{array}$ & $(\mathrm{GT})_{9}$ & $65^{\circ}$ & $196-226$ & 9 & 0.82 & 0.97 \\
\hline Dec13 & KT587700 & $\begin{array}{l}\text { F_CCCCCGTAAAACAGACAAAA } \\
\text { R_ACCATGATACAACGCTGCAA }\end{array}$ & $(T A)_{6}$ & $65^{\circ}$ & $166-178$ & 5 & 0.72 & 0.89 \\
\hline Dec14 & KT587701 & $\begin{array}{l}\text { F_AAACGGAGAAAGGGGATCAT } \\
\text { R_GAGCATACATGCAGCAGTGG }\end{array}$ & $(\mathrm{GAC})_{4}$ & $65^{\circ}$ & 290-310 & 3 & 0.62 & 0.22 \\
\hline Dec17 & KT587702 & $\begin{array}{l}\text { F_CCTTCGTCCATTACCCTGAA } \\
\text { R_ATCCACCAGTGCACGTATGA }\end{array}$ & $(\mathrm{TG})_{9}$ & $65^{\circ}$ & $224-248$ & 6 & 0.63 & 0.72 \\
\hline Dec18 & KT587703 & $\begin{array}{l}\text { F_ACGCACACACACGAACAAAT } \\
\text { R_ATTTCGACATGCCTGCAACT }\end{array}$ & $(\mathrm{CGAT})_{3}$ & $65^{\circ}$ & 180-202 & 6 & 0.78 & 0.96 \\
\hline Dec19 & KT587704 & $\begin{array}{l}\text { F_AGGTTCGATAATCGGCACAC } \\
\text { R_CGCAAGTGGTCAAGCAATTA }\end{array}$ & $(\mathrm{GT})_{7}$ & $65^{\circ}$ & $220-236$ & 6 & 0.79 & 0.95 \\
\hline Dec20 & KT587705 & $\begin{array}{l}\text { F_ACCTTGAACTCCTGCTTTTGT } \\
\text { R_AGCACTATCACCAATCAGCAA }\end{array}$ & $(A C)_{10}$ & $65^{\circ}$ & $150-168$ & 6 & 0.75 & 0.92 \\
\hline Dec21 & KT587706 & $\begin{array}{l}\text { F_GCCGACATCAACTTCCATTT } \\
\text { R_CTCCTTGGTCCAATTCCTCA }\end{array}$ & $(G T)_{7}$ & $65^{\circ}$ & 176-190 & 5 & 0.76 & 0.85 \\
\hline Dec22 & KT587707 & $\begin{array}{l}\text { F_GTGTGTACGTGATGCTATGTG } \\
\text { R_ATCGATCTCACTGACCATGT }\end{array}$ & $(\mathrm{CTT})_{4}$ & $65^{\circ}$ & 186-192 & 4 & 0.47 & 0.57 \\
\hline Dec24 & KT587708 & $\begin{array}{l}\text { F_TAAAGAAACATGGGCCGGTA } \\
\text { R_TTATTCCTGGGATTGGGTTG }\end{array}$ & $(\mathrm{GCC})_{5}$ & $65^{\circ}$ & $210-226$ & 5 & 0.73 & 0.86 \\
\hline Dec26 & KT587709 & $\begin{array}{l}\text { F_TCGGAAAACGCAGGAGAG } \\
\text { R_GTTCAGTGGGTCTGGCTTGT }\end{array}$ & $(\mathrm{CA})_{6}$ & $65^{\circ}$ & 180-190 & 4 & 0.68 & 0.59 \\
\hline Dec27 & KT587710 & $\begin{array}{l}\text { F_tGTACATGAATGGCAGCACA } \\
\text { R_AACAGCAGCAGAGATGACGA }\end{array}$ & $(\mathrm{AGAT})_{3}$ & $65^{\circ}$ & $248-262$ & 6 & 0.73 & 0.76 \\
\hline Dec28 & KT587711 & $\begin{array}{l}\text { F_GTTCCTCCCAAGAAACCACA } \\
\text { R_CCCAACATTCACCTGGTTCT }\end{array}$ & $(A C)_{6}$ & $65^{\circ}$ & $146-180$ & 8 & 0.78 & 0.84 \\
\hline Dec29 & KT587712 & $\begin{array}{l}\text { F_tGTTATAATCATCACCATGCTC } \\
\text { R_ACAGCTATTGCCACTACTTGA }\end{array}$ & $(\mathrm{GTA})_{4}$ & $65^{\circ}$ & $170-184$ & 6 & 0.70 & 0.67 \\
\hline Dec30 & KT587713 & $\begin{array}{l}\text { F_CATTACGAGCACGCAGTCC } \\
\text { R_TACCACTGCTGGACACGAGA }\end{array}$ & $(C A)_{7}$ & $65^{\circ}$ & $152-164$ & 5 & 0.71 & 0.59 \\
\hline Dec31 & KT587714 & $\begin{array}{l}\text { F_CGTTGTCAGCACACACACAC } \\
\text { R_TACTACCACTGCTGGACACGA }\end{array}$ & $(T C T A)_{3}$ & $65^{\circ}$ & $136-146$ & 5 & 0.70 & 0.79 \\
\hline
\end{tabular}


Table 2 continued

\begin{tabular}{|c|c|c|c|c|c|c|c|c|}
\hline SSR locus & $\begin{array}{l}\text { GenBank accession } \\
\text { number }\end{array}$ & Primer sequences $\left(5^{\prime}-3^{\prime}\right)$ & Repeat motif & $\mathrm{Ta}\left({ }^{\circ} \mathrm{C}\right)^{\mathrm{a}}$ & Size (bp) & $N A^{b}$ & $\mathrm{PIC}^{\mathrm{c}}$ & $D P^{d}$ \\
\hline Dec33 & KT587715 & $\begin{array}{l}\text { F_TGTCGTGTGCGTTTTGTTTT } \\
\text { R_CTAAGATCCCCACTCCCACA }\end{array}$ & $(\mathrm{CTT})_{4}$ & $60^{\circ}$ & $274-336$ & 8 & 0.78 & 0.94 \\
\hline Dec35 & KT587716 & $\begin{array}{l}\text { F_TTCTTGGACACACAGCCTTG } \\
\text { R_GGGCTGAAAACATCATCACC }\end{array}$ & $(\mathrm{TG})_{4}$ & $65^{\circ}$ & $274-290$ & 6 & 0.72 & 0.88 \\
\hline Dec36 & KT587717 & $\begin{array}{l}\text { F_GAAGGTGATGATCGGCAGTT } \\
\text { R_GTGTGCGTTGCTGCCTACTA }\end{array}$ & $(\mathrm{GCAG})_{3}$ & $65^{\circ}$ & 280 & 1 & 0.00 & 0.00 \\
\hline Dec37 & KT587718 & $\begin{array}{l}\text { F_CCTCTCTTCCGTTTGCTCTG } \\
\text { R_TGAACAGGCACGGATTGATA }\end{array}$ & $(\mathrm{GTG})_{5}$ & $65^{\circ}$ & $198-218$ & 5 & 0.70 & 0.81 \\
\hline Dec39 & KT587719 & $\begin{array}{l}\text { F_TAGGTGTCCCATTGGTCGAT } \\
\text { R_AGGAGAGCTGCGTGTCATTT }\end{array}$ & $(\mathrm{GT})_{7}$ & $55^{\circ}$ & $166-182$ & 5 & 0.64 & 0.34 \\
\hline Dec42 & KT587720 & $\begin{array}{l}\text { F_CACGTCATGTACTGCGATCC } \\
\text { R_GCGTCACACATACACACACG }\end{array}$ & $(\mathrm{GT})_{6}$ & $65^{\circ}$ & $220-230$ & 3 & 0.56 & 0.68 \\
\hline Dec43 & KT587721 & $\begin{array}{l}\text { F_CAGTCATCAGCATTCAGGTAT } \\
\text { R_ATAACTTGCGTATGTGCTCTC }\end{array}$ & $(\mathrm{TG})_{11}(\mathrm{AG})_{6}$ & $65^{\circ}$ & $212-228$ & 5 & 0.74 & 0.91 \\
\hline Dec44 & KT587722 & $\begin{array}{l}\text { F_CATGCTTAATCCAGAAATCAG } \\
\text { R_TGTAAACCGGAAAGTGTACTG }\end{array}$ & $(A C)_{12}$ & $65^{\circ}$ & $182-226$ & 6 & 0.78 & 0.94 \\
\hline Dec45 & KT587723 & $\begin{array}{l}\text { F_TGGAGATGGAGATGGGAGTC } \\
\text { R_CCCAAGGAATGGGATAGGTT }\end{array}$ & $(\mathrm{GGAT})_{3}$ & $65^{\circ}$ & 210 & 1 & 0.00 & 0.00 \\
\hline Dec47 & KT587724 & $\begin{array}{l}\text { F_AGAGAGCTGATGGTCGTGGT } \\
\text { R_TGGAAACTTGGGAGGATCTG }\end{array}$ & $(G A)_{9}$ & $65^{\circ}$ & 210 & 1 & 0.00 & 0.00 \\
\hline Dec48 & KT587725 & $\begin{array}{l}\text { F_CTAACGCTATTGCTTTGCTT } \\
\text { R_TGCAGAGAGAGAGAAGAGAGA }\end{array}$ & $(C T)_{45}$ & $65^{\circ}$ & 144-190 & 10 & 0.85 & 0.94 \\
\hline Dec49 & KT587726 & $\begin{array}{l}\text { F_CAATGCATGCTTGGAACTTG } \\
\text { R_CATCGGAGGGTAGATTGGTC }\end{array}$ & $(G T)_{6}$ & $65^{\circ}$ & $166-180$ & 5 & 0.65 & 0.74 \\
\hline Dec50 & KT587727 & $\begin{array}{l}\text { F_GAAACAGGACCATCAGATAGCA } \\
\text { R_GGAATCTGCAGGTTTGGAAG }\end{array}$ & $(C A)_{6}$ & $65^{\circ}$ & $164-180$ & 5 & 0.76 & 0.84 \\
\hline Dec51 & KT587728 & $\begin{array}{l}\text { F_GCTGATCCTCGGATTGTGTT } \\
\text { R_TAACTTGGACGCGCTAAAGG }\end{array}$ & $(\mathrm{TG})_{21}$ & $65^{\circ}$ & $248-262$ & 5 & 0.69 & 0.92 \\
\hline Dec52 & KT587729 & $\begin{array}{l}\text { F_CACGAATGCACATGCAATAA } \\
\text { R_AGTGAACCAAACTGCCAGAA }\end{array}$ & $(\mathrm{GT})_{6}$ & $65^{\circ}$ & $289-292$ & 2 & 0.00 & 0.00 \\
\hline Dec54 & KT587730 & $\begin{array}{l}\text { F_GCCCTCTTTAACTCTGCTTTA } \\
\text { R_GTATCTTCTTTCGGATGACCT }\end{array}$ & $(C A)_{8}$ & $65^{\circ}$ & $236-252$ & 5 & 0.75 & 0.92 \\
\hline Dec55 & KT587731 & $\begin{array}{l}\text { F_AGCACCATCATCTTTAACAAA } \\
\text { R_CAAGGAATTTGCACTAAAAGA }\end{array}$ & $(\mathrm{ACACC})_{3}$ & $65^{\circ}$ & $212-224$ & 6 & 0.78 & 0.73 \\
\hline Dec56 & KT587732 & $\begin{array}{l}\text { F_GAACTTAATGGCGGAGTAGAC } \\
\text { R_CACAGATTGCTGAATTGTTTC }\end{array}$ & $(\mathrm{AG})_{14}$ & $55^{\circ}$ & $220-230$ & 2 & 0.00 & 0.00 \\
\hline Dec58 & KT587733 & $\begin{array}{l}\text { F_ATTAGGATTGCGCACTGGTC } \\
\text { R_ATCCGCATTCACAACCTCTC }\end{array}$ & $(G T)_{6}$ & $65^{\circ}$ & $286-298$ & 5 & 0.64 & 0.8 \\
\hline Dec59 & KT587734 & $\begin{array}{l}\text { F_GGTTAAAATGGTTCGCTGGA } \\
\text { R_ACCTAGGCTCGCATGACAAT }\end{array}$ & $(\mathrm{GT})_{7}$ & $65^{\circ}$ & $184-220$ & 5 & 0.73 & 0.92 \\
\hline Dec60 & KT587735 & $\begin{array}{l}\text { F_ATTTCAGTTGCACATTCCA } \\
\text { R_TCCAAAACTTAGCTCAGAAAG }\end{array}$ & $(\mathrm{GT})_{6}$ & $55^{\circ}$ & $220-230$ & 2 & 0.00 & 0.00 \\
\hline Dec62 & KT587736 & $\begin{array}{l}\text { F_AGGAAGGGTACGGTGTAGGC } \\
\text { R_TCTACATGCACATCCGGAAA }\end{array}$ & $(\mathrm{CA})_{7}$ & $65^{\circ}$ & $216-238$ & 4 & 0.41 & 0.59 \\
\hline Dec63 & KT587737 & $\begin{array}{l}\text { F_GGGATATTTTCCGGATGT } \\
\text { R_CAGAGCTCAGAAAGTCGTTAC }\end{array}$ & $(\mathrm{CTT})_{4}$ & $65^{\circ}$ & $218-226$ & 3 & 0.51 & 0.7 \\
\hline Dec65 & KT587738 & $\begin{array}{l}\text { F_TCGGATTCTTGGACAACCTC } \\
\text { R_CCTCTACGCGAAAGATGGTC }\end{array}$ & $(\mathrm{GGCC})_{3}$ & $65^{\circ}$ & 180 & 1 & 0.00 & 0.00 \\
\hline
\end{tabular}


Table 2 continued

\begin{tabular}{|c|c|c|c|c|c|c|c|c|}
\hline SSR locus & $\begin{array}{l}\text { GenBank accession } \\
\text { number }\end{array}$ & Primer sequences $\left(5^{\prime}-3^{\prime}\right)$ & Repeat motif & $\operatorname{Ta}\left({ }^{\circ} \mathrm{C}\right)^{\mathrm{a}}$ & Size (bp) & $N A^{b}$ & PIC $^{c}$ & $D P^{d}$ \\
\hline \multirow[t]{2}{*}{ Dec69 } & KT587739 & F_GATGGCTACCTGCATTGGAT & $(\mathrm{CCAT})_{3}$ & $55^{\circ}$ & $168-180$ & 6 & 0.79 & 0.96 \\
\hline & & R_ATAAGGGGAGCCCTCAAAAA & & & & & & \\
\hline \multirow[t]{2}{*}{ Dec70 } & KT587740 & F_AGCTGCCTCCACTTGACAAT & $(\mathrm{TG})_{7}$ & $65^{\circ}$ & $256-268$ & 5 & 0.72 & 0.62 \\
\hline & & R_AGGCCCTGATAGTCCCCTAA & & & & & & \\
\hline \multirow[t]{2}{*}{ Dec71 } & KT587741 & F_GAGCTTCCCTGTGTCTGATA & $(\mathrm{TG})_{10}$ & $55^{\circ}$ & $234-254$ & 4 & 0.62 & 0.84 \\
\hline & & R_ATGACAATGACTATGCTGACC & & & & & & \\
\hline \multirow[t]{2}{*}{ Dec75 } & KT587742 & F_ACAGGAGCCTTTATGCATGG & $(\mathrm{ATGC})_{3}$ & $65^{\circ}$ & $150-166$ & 5 & 0.68 & 0.69 \\
\hline & & R_GTCCTGTGTTGGTCGTTCCT & & & & & & \\
\hline \multirow[t]{2}{*}{ Dec76 } & KT587743 & F_GTCACGTGCCATCACAAATC & $(\mathrm{TAGC})_{3}$ & $65^{\circ}$ & 270 & 1 & 0.00 & 0.00 \\
\hline & & R_GCACACATGCATGATGACAA & & & & & & \\
\hline \multirow[t]{2}{*}{ Dec77 } & KT587744 & F_TCCAAATGTACCGTCAATAAA & $(A G)_{12}$ & $55^{\circ}$ & $234-260$ & 7 & 0.76 & 0.9 \\
\hline & & R_CGTGTCTGCATTCAAAGTG & & & & & & \\
\hline \multirow[t]{2}{*}{ Dec78 } & KT587745 & F_GCTTACCACATCCGGTGATT & $(\mathrm{AC})_{8}$ & $65^{\circ}$ & $246-260$ & 5 & 0.66 & 0.71 \\
\hline & & R_GAGAATGCTTCCCGTTCTTG & & & & & & \\
\hline \multirow[t]{2}{*}{ Dec83 } & KT587746 & F_GGCTTGCTCCAAGAGATGAG & $(C A)_{20}$ & $65^{\circ}$ & 174-198 & 4 & 0.66 & 0.72 \\
\hline & & R_TAGCTTGGCCTTTGTGTGTG & & & & & & \\
\hline \multirow[t]{2}{*}{ Dec84 } & KT587747 & F_GGCTTGCTCCAAGAGATGAG & $(\mathrm{AC})_{9}$ & $65^{\circ}$ & $220-250$ & 7 & 0.78 & 0.95 \\
\hline & & R_TTCGTCACGTCAAAACAAGC & & & & & & \\
\hline \multirow[t]{2}{*}{ Dec86 } & KT587748 & F_CCACCTCCCAGGATAGATGA & $(\mathrm{TG})_{7}$ & $55^{\circ}$ & $140-180$ & 9 & 0.80 & 0.94 \\
\hline & & R_AGATTGGGGGAGGAAGAAGA & & & & & & \\
\hline \multirow[t]{2}{*}{ Dec89 } & KT587749 & F_CTGTTGCATCCACCACTTTTT & $(\mathrm{TC})_{8}$ & $65^{\circ}$ & $146-180$ & 4 & 0.55 & 0.41 \\
\hline & & R_CGGCAGCCTAAAGTGATTGT & & & & & & \\
\hline \multirow[t]{2}{*}{ Dec90 } & KT587750 & F_CGGTGCTCCATGATTAGGAT & $(\mathrm{GT})_{8}$ & $65^{\circ}$ & $278-326$ & 7 & 0.77 & 0.82 \\
\hline & & R_GCGTAGCATCATCGAGAACA & & & & & & \\
\hline \multirow[t]{2}{*}{ Dec91 } & KT587751 & F_GCCTCATCTGTTCATTCATT & $(\mathrm{TG})_{7}$ & $55^{\circ}$ & 290-330 & 3 & 0.26 & 0.22 \\
\hline & & R_TGGCACTCTAACTTGTAGGC & & & & & & \\
\hline \multirow[t]{2}{*}{ Dec92 } & KT587752 & F_AGCAATCCAAGCTGAAAGGA & $(\mathrm{AC})_{7}$ & $65^{\circ}$ & $264-290$ & 7 & 0.79 & 0.92 \\
\hline & & R_TTCCGCATGAAACAAAACTG & & & & & & \\
\hline \multirow[t]{2}{*}{ Dec93 } & KT587753 & F_TTCGGTCAAAATCGAAAAGG & $(A C)_{6}$ & $65^{\circ}$ & $226-244$ & 5 & 0.72 & 0.95 \\
\hline & & R_GCATTGTTTCAGAGGCTTCG & & & & & & \\
\hline \multirow[t]{2}{*}{ Dec95 } & KT587754 & F_AGCAACCCAAAGGTCAGCTA & $(C T)_{24}$ & $65^{\circ}$ & $178-208$ & 6 & 0.71 & 0.89 \\
\hline & & R_AGGAGGGATTCAAGGGAGAA & & & & & & \\
\hline \multirow[t]{2}{*}{ Dec96 } & KT587755 & F_CATTCTGGTATGGCACGTTG & $(C A)_{6}$ & $65^{\circ}$ & $148-154$ & 4 & 0.66 & 0.85 \\
\hline & & R_ATTTACCGACCAGGCTGAAG & & & & & & \\
\hline \multirow[t]{2}{*}{ Dec97 } & KT587756 & F_GGGCAGGCACTAGATTGATT & $(\mathrm{TCTT})_{3}$ & $65^{\circ}$ & $176-184$ & 4 & 0.61 & 0.72 \\
\hline & & R_TTGCTTGCTTGAGTTTGTGG & & & & & & \\
\hline \multirow[t]{2}{*}{ Dec98 } & KT587757 & F_TAGGTGACAAGGCACGATCA & $(A G)_{10}$ & $65^{\circ}$ & $252-272$ & 7 & 0.76 & 0.95 \\
\hline & & R_GGGCCAACATACCAAAGAGA & & & & & & \\
\hline \multirow[t]{2}{*}{ Dec99 } & KT587758 & F_TAAGAGACGAGTGCTCTGAAA & $(\mathrm{AGCAGG})_{3}$ & $65^{\circ}$ & $210-228$ & 7 & 0.77 & 0.91 \\
\hline & & R_TTGTGAATCGGTACTTTTGTC & & & & & & \\
\hline Dec101 & KT587759 & F_CTCTAACTTTCGGCGTGGTC & $(\mathrm{GGCC})_{3}$ & $65^{\circ}$ & $224-230$ & 3 & 0.53 & 0.71 \\
\hline & & R_GGACGGTCCGACTTGTCTAA & & & & & & \\
\hline Dec103 & KT587760 & F_ATGACGAACTTGCTCCCTACA & $(A C)_{8}$ & $55^{\circ}$ & $176-206$ & 4 & 0.51 & 0.71 \\
\hline & & R_ATCGATTCAGAGCCGCTTC & & & & & & \\
\hline Dec105 & KT587761 & F_CCTTCTGTTCATTGCAGTCC & $(\mathrm{TG})_{8}$ & $65^{\circ}$ & $174-180$ & 4 & 0.56 & 0.65 \\
\hline & & R_TGGTACCACAATGCCAAATC & & & & & & \\
\hline Dec106 & KT587762 & F_TCACGAACAACGATCAGAGC & $(\mathrm{TG})_{7}$ & $55^{\circ}$ & $180-230$ & 7 & 0.74 & 0.93 \\
\hline & & R_TCTTTACCCGTGCTGTTTCC & & & & & & \\
\hline
\end{tabular}


Table 2 continued

\begin{tabular}{|c|c|c|c|c|c|c|c|c|}
\hline SSR locus & $\begin{array}{l}\text { GenBank accession } \\
\text { number }\end{array}$ & Primer sequences $\left(5^{\prime}-3^{\prime}\right)$ & Repeat motif & $\mathrm{Ta}\left({ }^{\circ} \mathrm{C}\right)^{\mathrm{a}}$ & Size (bp) & $N A^{b}$ & PIC $^{c}$ & $D P^{d}$ \\
\hline Dec108 & KT587763 & $\begin{array}{l}\text { F_CATCACCGCATTTATGCAAG } \\
\text { R_ACACACGTCCTCGTCTTCCT }\end{array}$ & $(A G)_{8}$ & $65^{\circ}$ & $184-200$ & 6 & 0.68 & 0.85 \\
\hline Dec109 & KT587764 & $\begin{array}{l}\text { F_CAGCACACTGAATCCTCTGC } \\
\text { R_CCGTTGTTCCATCAGAACCT }\end{array}$ & $(\mathrm{GT})_{6}$ & $65^{\circ}$ & $216-220$ & 3 & 0.39 & 0.59 \\
\hline Dec110 & KT587765 & $\begin{array}{l}\text { F_CTCCGAAGATCCGAGCTATG } \\
\text { R_CCCCTGGAGGCTATAAAAGG }\end{array}$ & $(\mathrm{GT})_{7}$ & $65^{\circ}$ & $178-184$ & 4 & 0.31 & 0.41 \\
\hline Dec111 & KT587766 & $\begin{array}{l}\text { F_tGATTAGGTGCTGACTGCTG } \\
\text { R_CTGGAAGATGTATTTGGTGTGA }\end{array}$ & $(\mathrm{ATTT})_{3}$ & $65^{\circ}$ & 178-186 & 5 & 0.50 & 0.57 \\
\hline Dec112 & KT587767 & $\begin{array}{l}\text { F_CCTCAAGAAGCTCTGGGATTT } \\
\text { R_TGTGCAAACGTCAGTAGAGCA }\end{array}$ & $(T G T T)_{3}$ & $55^{\circ}$ & $238-244$ & 4 & 0.57 & 0.72 \\
\hline Dec113 & KT587768 & $\begin{array}{l}\text { F_tGGACTAACTGCACTGCCTGT } \\
\text { R_CATGAGGAGCACAGCGAATA }\end{array}$ & $(\mathrm{GT})_{9}$ & $65^{\circ}$ & $208-224$ & 7 & 0.74 & 0.94 \\
\hline Dec114 & KT587769 & $\begin{array}{l}\text { F_CAAAGGCCATGCCTTGTACT } \\
\text { R_CACTGCTCAGCCAATCCTAAG }\end{array}$ & $(G T)_{11}$ & $65^{\circ}$ & $214-220$ & 4 & 0.62 & 0.72 \\
\hline Dec115 & KT587770 & $\begin{array}{l}\text { F_GGCATATGTCTGAGTAAGTGTG } \\
\text { R_CCTGTTTCCATTGATTCTTTTT }\end{array}$ & $(\mathrm{TCT})_{4}$ & $55^{\circ}$ & $160-174$ & 6 & 0.76 & 0.6 \\
\hline Dec116 & KT587771 & $\begin{array}{l}\text { F_tCACTTCATCCATTCGCTTG } \\
\text { R_AACATGACCGACTCCTACGG }\end{array}$ & $(\mathrm{TG})_{17}$ & $65^{\circ}$ & 274 & 1 & 0.00 & 0.00 \\
\hline Dec118 & KT587772 & $\begin{array}{l}\text { F_ACACACCCCAACTCACACAA } \\
\text { R_TGGTCATGGCAAAAGATGAA }\end{array}$ & $(A C)_{6}$ & $65^{\circ}$ & $208-226$ & 6 & 0.75 & 0.83 \\
\hline Dec121 & KT587773 & $\begin{array}{l}\text { F_TGCACAATGATGAACACAGG } \\
\text { R_AGTGAACCAAACTGCCAGAA }\end{array}$ & $(\mathrm{GT})_{7}$ & $65^{\circ}$ & $226-264$ & 6 & 0.74 & 0.74 \\
\hline Dec122 & KT587774 & $\begin{array}{l}\text { F_CCTGCGTCACTCGAGAAAA } \\
\text { R_CAATGTCATCGCCATTTCTG }\end{array}$ & $(\mathrm{TCTG})_{3}$ & $65^{\circ}$ & $268-292$ & 6 & 0.76 & 0.93 \\
\hline Dec123 & KT587775 & $\begin{array}{l}\text { F_tGAGCAACACTGGAGAATGG } \\
\text { R_CGTACATGACAGGAGGGTGTT }\end{array}$ & $(\mathrm{TC})_{9}$ & $65^{\circ}$ & $248-280$ & 9 & 0.80 & 0.94 \\
\hline Dec124 & KT587776 & $\begin{array}{l}\text { F_AGAAGCCCCAGATGTTCTGA } \\
\text { R_GCTAGTCGCGTCTACCGTTC }\end{array}$ & $(\mathrm{GT})_{9}$ & $65^{\circ}$ & $270-306$ & 4 & 0.52 & 0.69 \\
\hline Dec125 & KT587777 & $\begin{array}{l}\text { F_TCTGGGGTGGAAATGTTGAT } \\
\text { R_CCCTTCACCTTGAGAAAGCA }\end{array}$ & $(\mathrm{CT})_{11}$ & $65^{\circ}$ & $202-214$ & 4 & 0.61 & 0.34 \\
\hline Dec126 & KT587778 & $\begin{array}{l}\text { F_GGATGGATTGATGGATGCTT } \\
\text { R_AACCCGAAAGGCCTAAGCTA }\end{array}$ & $(\mathrm{GGCC})_{3}$ & $65^{\circ}$ & $268-304$ & 7 & 0.77 & 0.93 \\
\hline Dec127 & KT587779 & $\begin{array}{l}\text { F_CGTTGATCACACGTCTCAGG } \\
\text { R_GATTTCGCCACCAACATTCT }\end{array}$ & $(\mathrm{TTGC})_{3}$ & $65^{\circ}$ & $250-280$ & 4 & 0.65 & 0.75 \\
\hline Dec131 & KT587780 & $\begin{array}{l}\text { F_CTTGTTACCTTCTGCACAATAAA } \\
\text { R_ATTAGTCTTTCCGTCCTTGTC }\end{array}$ & $(\mathrm{GAA})_{5}$ & $65^{\circ}$ & $160-170$ & 3 & 0.00 & 0.00 \\
\hline Dec132 & KT587781 & $\begin{array}{l}\text { F_GTATCGGGTAGCAAGGCAAG } \\
\text { R_GGAAATTCCTTACCCCGAAG }\end{array}$ & $(A A G C)_{3}$ & $65^{\circ}$ & $220-240$ & 2 & 0.00 & 0.00 \\
\hline Dec133 & KT587782 & $\begin{array}{l}\text { F_GGATGGAAGAGCACAAAAGC } \\
\text { R_GCGTGTGTGTGTGTGTTTGA }\end{array}$ & $(\mathrm{CT})_{7}$ & $65^{\circ}$ & $218-228$ & 5 & 0.68 & 0.81 \\
\hline Dec134 & KT587783 & $\begin{array}{l}\text { F_CAGGCTTCCCCTCTCTCTCT } \\
\text { R_GCAACCGGAAGAATTCATGT }\end{array}$ & $(A C)_{7}$ & $65^{\circ}$ & $220-260$ & 8 & 0.76 & 0.93 \\
\hline Total average & & & & & & 4.93 & 0.68 & 0.77 \\
\hline
\end{tabular}

${ }^{a}$ Amplification temperature $\left({ }^{\circ} \mathrm{C}\right)$

${ }^{b}$ Maximum number of alleles observed

c Polymorphism information content

d Discrimination power 


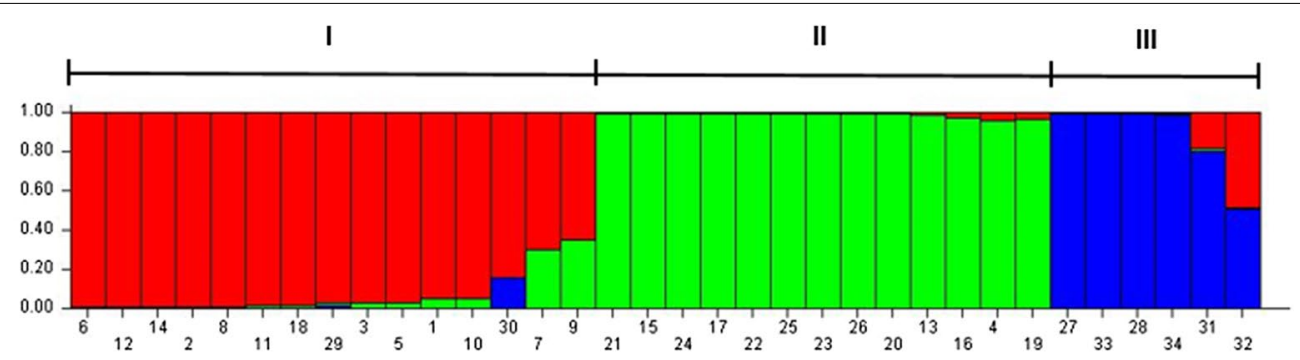

Fig. 1 Analysis performed using an admixture model in STRUCTURE 2.3.4 software with correlated allele frequencies. The clustering profile obtained at $\mathrm{K}=3$ is indicated by different colors. Each of the 34 genotypes is represented by a single column broken into colored segments with lengths proportional to each of the K inferred gene pools. The scale on the left indicates the membership coefficients (Q) used to allocate the genotypes into clusters. The genotypes were named according to the annotated numbers listed in Table 1. Cluster I (from 6 to 9), Cluster II (from 21 to 19) and Cluster III (from 27 to 32)

(>20 bp), and $78 \%$ were classified as class II motifs (from 12 to $20 \mathrm{bp}$ ).

A total of 113 specific primer pairs were designed, and 93 SSR markers amplified from $U$. decumbens, with 82 of these being polymorphic. A total of 459 bands were scored, and the number of bands per locus was found to range from 1 to 10 , with an average of 4.93 bands per locus (Table 2).

The PIC values of the 82 polymorphic loci ranged from 0.26 to 0.85 (average of 0.68 ), and the discrimination power (DP) values ranged from 0.22 to 0.97 (average of 0.77) (Table 2).

Two genotypes of four other species of the genus Urochloa (U. brizantha, $U$. humidicola, $U$. dictyoneura and $U$. ruziziensis) (Table 1) were used to evaluate the transferability of the 93 SSR markers. All of the loci were tested using the same PCR conditions used for analysis of $U$. decumbens. Fifty-six percent of the loci were amplified in at least one $U$. dictyoneura genotype, $38 \%$ were amplified in $U$. humidicola, 99 \% were amplified in $U$. ruziziensis, and $92 \%$ were amplified in $U$. brizantha. Amplification of $33 \%$ of the microsatellite markers was achieved for all of the evaluated species. The microsatellite markers Dec07, Dec31, Dec33, Dec77 and Dec108 were only transferable for $U$. ruziziensis species (see Additional file 1).

Based on the allelic frequencies determined using STRUCTURE software [23], $28 \%$ of the alleles are rare (frequency $<0.05$ ), $57 \%$ of these alleles are of intermediate abundance $(0.05<$ frequency $<0.30)$, and $15 \%$ are abundant alleles (frequency $>0.30$ ). We observed 43 rare alleles that are specific for $U$. decumbens, eight rare alleles specific for $U$. humidicola, seven specific for $U$. dictyoneura, four alleles specific for $U$. brizantha and two rare alleles specific for $U$. ruziziensis.

The Bayesian analysis performed using STRUCTURE software [23] revealed that the 34 Urochloa genotypes could be distributed into three distinct clusters (Fig. 1), as determined from the $\Delta \mathrm{K}$ values that were generated using Structure Harvester software [24, 25] (see Additional file 2). Using a $K$ value of three, 15 genotypes were allocated into Cluster I (6 to 9), 13 genotypes were grouped into Cluster II (21 to 19) and six genotypes were allocated into Cluster III (27 to 32) (Fig. 1).

The genetic distance values that were determined using Jaccard's coefficient ranged from 0.06 (D062 and R10) to 0.76 (H016 and D009) (see Additional file 3). The unrooted neighbor-joining tree successfully discriminated all of the tested genotypes (Fig. 2).

\section{Discussion}

In this report, we have described the first set of microsatellite markers for $U$. decumbens, which is an important tropical forage grass for which there is limited genetic information. The availability of a robust set of informative molecular markers is essential to accelerating its breeding programs as well as for germplasm characterization, genetic map development and marker-assisted selection.

In the present study, dinucleotide repeats were the most abundant class of microsatellites detected, followed by tetra, tri, mono, hexa and pentanucleotide repeats. Dinucleotide motifs have been found to be the most abundant type of microsatellites in plant genomes [28, 29]. Notably, the high occurrence of dinucleotide motifs can be attributed to both of the evaluated libraries having been enriched using $(\mathrm{CT})_{8}$ and $(\mathrm{GT})_{8}$ probes.

In total, 93 SSR markers were characterized, 82 of which were found to be polymorphic (88 \%). The loci that did not exhibit polymorphism in the genotypes that we evaluated may be useful in other studies.

The Polymorphism Information Content (PIC) is an index used to qualify a marker for genetic studies and reflects the level of polymorphism detected. Seventy-seven markers tested in $U$. decumbens genotypes were found to be highly informative (PIC > 0.5) and 


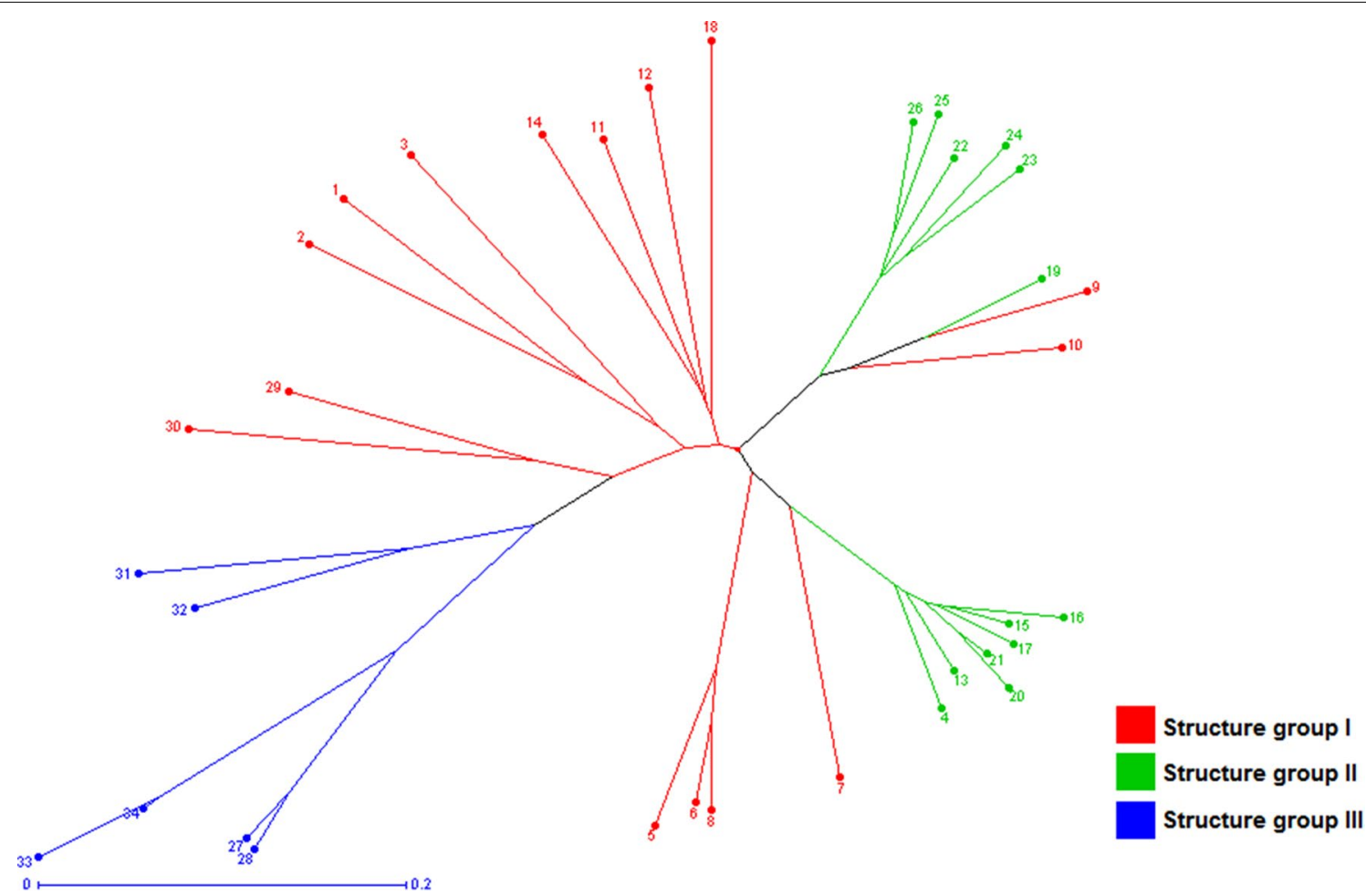

Fig. 2 Unrooted neighbor-joining tree based on Jaccard's coefficient for the 34 genotypes of the Urochloa species. The genotypes were named according to the annotated numbers listed in Table 1. The colors of the branches represent the clusters identified in Fig. 1, as follows: red Cluster I; green Cluster II; blue Cluster III

five markers were found to be moderately informative $(0.25<$ PIC $<0.5)$, based on a previously proposed classification system [30] (Table 2). The Dec48 marker had the highest PIC value, 0.85 , and the Dec91 marker had the lowest value, 0.26 . The average PIC values for all of the markers was 0.68 (Table 2), indicating a high level of polymorphism.

To determine whether these molecular markers could discriminate the genotypes of $U$. decumbens, the discrimination power (DP) of each SSR locus was computed. The PD values ranged from 0.22 (Dec14 and Dec91) to 0.97 (Dec12), with an average value of 0.77 .

The most informative loci in this panel of SSRs were Dec12, Dec48, Dec86 and Dec97 because they had the highest PIC and DP values (Table 2). In contrast, the Dec91 locus had low PIC and DP values $(0.26$ and 0.22 , respectively), as expected due to its low levels of polymorphism and cross- amplification in all of the other Urochloa species tested, which suggests that this locus is a conserved region [11].

Structure analysis showed that the genotypes were distributed in three clusters and that each cluster was characterized by a set of allele frequencies at each locus and was represented by different colors (red, green and blue) as shown in Fig. 1. The best $\mathrm{K}$ number of clusters was determined using the $\Delta \mathrm{K}$ method [24] and implemented in the online tool Structure Harvester [25] (see Additional file 2).

Cluster I included fifteen $U$. decumbens genotypes plus the $U$. ruziziensis genotypes, Cluster II contained only $U$. decumbens genotypes, and Cluster III contained the others Urochloa species, including $U$. dictyoneura, $U$. humidicola and $U$. brizantha (Fig. 1). The clustering of some of the $U$. decumbens genotypes with $U$. ruziziensis genotypes may be explained by the genetic proximity of these species [11, 13, 31, 32]. This fact is reflected in the allelic pools that are identified with different colors in Fig. 1.

Cluster II included genotypes 19 and 20, and six hybrids derived from crosses between these two genotypes that were grouped together (Fig. 1). These hybrids are members of an $F_{1}$ population that will be mapped using the polymorphic SSRs described in this study. In Cluster III, which included three different Urochloa species, the predominant allelic pool is represented in blue, and only the $U$. brizantha genotypes showed some percentage of the red allelic pools, demonstrating their genetic proximity to $U$. decumbens (Fig. 1).

The tree constructed based on Jaccard's coefficient successfully discriminated all of the tested genotypes (Fig. 2) 
and showed a distribution of these genotypes similar to that obtained using STRUCTURE software [23] (Fig. 1), although the two types of analysis used different statistical approaches. Moreover, this tree and the allelic pools that were determined indicated that $U$. decumbens and $U$. ruziziensis are more closely related to one another than to the other species (Figs. 1 and 2).

Based on the genetic values obtained using Jaccard's coefficient, the lowest genetic distance was observed between the D062 and R10 genotypes (0.06). The R10 genotype should correspond to a hybrid that originated from a cross between D062 and D24/27, but the genetic distance observed shows that it is likely a false hybrid, which demonstrates the importance of using molecular markers to discriminate genotypes. The highest genetic distance $(0.76)$ was observed between the D009 and H016 genotypes, representing $U$. decumbens and $U$. humidicola species, respectively, which are genetically distant species $[11,13,31,32]$ (see Additional file 3).

All of the microsatellite markers were transferable to at least one different species of the Urochloa genus, and $33 \%$ of the markers were successfully amplified in all of the species, indicating their absolute transferability. The highest level of transferability was observed in $U$. ruziziensis, followed by $U$. brizantha, $U$. dictyoneura and $U$. humidicola (see Additional file 1). The higher proportion of successful PCR amplification for the $U$. ruziziensis and $U$. brizantha genotypes indicates the closer phylogenetic distance between these species and $U$. decumbens. Thus, $U$. brizantha, $U$. decumbens and $U$. ruziziensis form an agamic complex and produce fertile hybrids [33, 34], enhancing the Urochloa breeding program.

Silva et al. [12] developed 198 polymorphic microsatellite markers for $U$. ruziziensis and found that the percentages of markers potentially transferable to $U$. decumbens and $U$. humidicola were $92.9 \%$ and $42.9 \%$, respectively, corroborating our results. Others studies showed that $U$. brizantha and $U$. ruziziensis are more closely related to $U$. decumbens than to $U$. humidicola and $U$. dictyoneura [11, 13, 31, 32]. Marker transferability is effective in reducing the time and cost of initial studies aimed at identifying microsatellite markers in related species; thus, these markers could be used in genetics studies, such as in those concerning intra-species molecular characterization, species differentiation, molecular identification, and characterization of interspecific hybrids [14].

The success of a breeding program can be accelerated by the effective use of molecular markers. Thus, the SSR markers developed in this study will be useful for $U$. decumbens breeding programs and possibly for those of other related Urochloa species.

\section{Availability of supporting data}

The datasets supporting the results of this article are included in the article.

\section{Additional files}

Additional file 1. Transferability of the SSR markers developed for Urochloa decumbens.

Additional file 2. Magnitude of $\triangle K$ determined in STRUCTURE analysis of $K$, calculated following the $\Delta K$ method proposed by Evanno et al. [24].The highest $\Delta K$ value corresponds to the optimal $K$.

Additional file 3. Jaccard's coefficient for 34 genotypes of Urochloa spp. evaluated using 82 microsatellite markers. Individuals are identified according to their EBC codes (Table 1).

\section{Abbreviations}

AN: annotation number; bp: base pairs; CAPES: Coordination of Improvement of Higher Education Personnel; CTAB: cetyltrimethyl ammonium bromide; DNA: deoxyribonucleic acid; DP: discrimination power; EBC: Embrapa Beef Cattle; EMBRAPA: Brazilian Agricultural Research Corporation; K: number of clusters; MCMC: Markov Chain Monte Carlo; NA: number of alleles; NJ: neighbor joining; PCR: polymerase chain reaction; PIC: polymorphism information content; Q: association coefficient determined using STRUCTURE analysis; QTL: quantitative trait loci; SSR: simple sequence repeat; Syn: synonym; Ta $\left({ }^{\circ} \mathrm{C}\right)$ : annealing temperature.

\section{Authors' contributions}

LJC developed the microsatellite-enriched libraries. RCUF conducted the bioinformatics searches to identify the microsatellites, designed the flanking primers, validated the microsatellite markers, performed the statistical analysis and drafted the manuscript. CBV and LC participated in the design and implementation of the study. LC helped draft the manuscript. APS conceived and supervised the study and helped to draft the manuscript. All authors read and approved the final manuscript.

\section{Author details}

${ }^{1}$ Centro de Biologia Molecular e Engenharia Genética (CBMEG), Universidade Estadual de Campinas (UNICAMP), Cidade Universitária Zeferino Vaz, CP 6010 Campinas, SP CEP 13083-875, Brazil. ² EMBRAPA Genetic Resources and Biotechnology, Brazilian Agricultural Research Corporation, CP 02372, Brasília, DF CEP 70770-900, Brazil. ${ }^{3}$ EMBRAPA Beef Cattle, Brazilian Agricultural Research Corporation, CP 154, Campo Grande, MS CEP 79002-970, Brazil. ${ }^{4}$ Departamento de Biologia Vegetal, Instituto de Biologia, Universidade Estadual de Campinas (UNICAMP), Cidade Universitária Zeferino Vaz, CP 6109, Campinas, SP CEP 13083-862, Brazil.

\section{Acknowledgements}

The authors thank the Fundação de Amparo à Pesquisa de SP (FAPESP 08/52197-4) and the Coordenação de Aperfeiçoamento de Pessoal de Nível Superior (CAPES - Computational Biology Program) for grants; the Brazilian Agricultural Research Corporation (EMBRAPA Beef Cattle) for providing the Urochloa genotypes used. RCUF is a recipient of a graduate fellowship from CAPES-EMBRAPA Program.

\section{Competing interests}

The authors declare that they have no competing interests.

Received: 2 October 2015 Accepted: 1 March 2016

Published online: 10 March 2016

\section{References}

1. Associação Brasileira das Indústrias Exportadoras de Carne (ABIEC). Estatísticas: balanço da pecuária. http://www.abiec.com.br/texto. asp?id=8. 2014. Accessed 15 July 2015 . 
2. Valle $C B$, Jank $L$, Resende RMS. O melhoramento de forrageiras tropicais no Brasil. Rev. Ceres. 2009;56(4):460-72.

3. Jank L, do Valle CB, Resende RMS. Breeding tropical forage. Crop Breed Appl Biotechnol. 2011;S1:27-34.

4. Mateus RG, Barrios SCL, Figueiredo UJ, do Valle CB. Agronomic evaluation of 324 intraspecific hybrids of Brachiaria decumbens in Brazil. Trop Grasslands Forrajes Tropicales. 2013;1:99-100.

5. Penteado MIO, dos Santos ACM, Rodrigues IF, do Valle CB, Seixas MAC, Esteves A. Determinação de ploidia e avaliação da quantidade de DNA total em diferentes espécies do gênero Brachiaria. 2000. http://www. embrapa.br/gado-de-corte/busca-de-publicacoes/-/publicacao/324069/ determinacao-de-ploidia-e-avaliacao-da-quantidade-de-dna-total-emdiferentes-especies-do-genero-brachiaria. Accessed 30 July 2015.

6. Kalia RK, Rai MK, Kalia S, Singh R, Dhawan AK. Microsatellite markers: an overview of the recent progress in plants. Euphytica. 2011. doi:10.1007/ s10681-010-0286-9.

7. Gao C, Ren X, Mason AS, Li J, Wang W, Xiao M, Fu D. Revisiting an important component of plant genomes: microsatellites. Funct Plant Biol. 2013. doi:10.1071/FP12325.

8. Haq SU, Jain R, Sharma M, Kachhwaha S, Kothari SL. Identification and characterization of microsatellites in expressed sequence tags and their cross transferability in different plants. Int J Genomics. 2014. doi:10.1155/2014/863948.

9. Jungmann L, Vigna BBZ, Paiva J, Sousa ACB, do Valle CB, Laborda PR, Zucchi MI, de Souza AP. Development of microsatellite markers for Brachiaria humidicola (Rendle) Schweick. Conserv Genet Resour. 2009. doi:10.1007/ s12686-009-9111-y.

10. Jungmann L, Sousa ACB, Paiva J, Francisco PM, Vigna BBZ, do Valle CB, Zucchi MI, de Souza AP. Isolation and characterization of microsatellite markers for Brachiaria brizantha (Hochst. ex A. Rich.) Stap. Conserv Genet. 2009. doi:10.1007/s10592-009-9839-7.

11. Vigna BBZ, Alleoni GC, Jungmann $L$, do Valle CB, de Souza AP. New microsatellite markers developed from Urochloa humidicola (Poaceae) and cross amplification in different Urochloa species. BMC Res Notes. 2011:4:523.

12. Silva PIT, Martins AM, Gouvea EG, Pessoa-Filho M, Ferreira ME. Development and validation of microsatellite markers for Brachiaria ruziziensis obtained by partial genome assembly of Illumina single-end reads. BMC Genom. 2013. doi:10.1186/1471-2164-14-17.

13. Santos JCS, Barreto MA, Oliveira FA, Vigna BBZ, Souza AP. Microsatellite markers for Urochloa humidicola (Poaceae) and their transferability to other Urochloa species. BMC Res Notes. 2015;8:83.

14. Azevedo ALS, Costa PP, Machado JC, Machado MA, Pereira AV, Lédo FJS. Cross species amplification of Pennisetum glaucum microsatellite markers in Pennisetum purpureum and genetic diversity of napier grass accessions. Crop Sci. 2012. doi:10.2135/cropsci2011.09.0480.

15. Doyle JJ, Doyle JL. A rapid DNA isolation procedure for small quantities of fresh leaf tissue. Phytochem Bull. 1987;19:11-5.

16. Billotte N, Lagoda PJL, Risterucci AM, Baurens FC. Microsatellite-enriched libraries: applied methodology for the development of SSR markers in tropical crops. Fruits. 1999:54:277-88.

17. Thiel T. MISA: MIcroSAtellite identification tool. Version 1.0. In: MISAMlcroSAtellite identification tool. Leibniz Institute of Plant Genetics and Crop Plant Research. 2001. http://pgrc.ipkgatersleben.de/misa/misa.html. Accessed 15 August 2014
18. Untergasser A, Nijveen H, Rao X, Bisseling T, Geurts R, Leunissen JA. Primer3Plus, an enhanced web interface to Primer3. Nucleic Acids Res. 2007. doi:10.1093/nar/gkm306.

19. Creste S, Tulmann Neto A, Figueira A. Detection of single sequence repeat polymorphisms in denaturing polyacrylamide sequencing gels by silver staining. Plant Mol Bio Rep. 2001. doi:10.1007/BF02772828.

20. Jaccard P. Nouvelles recherches sur la distribution florale. Bull Soc Vaudoise Sci Nat. 1908;44:223-70.

21. Rohlf FJ. NTSYSpc: numerical taxonomy and multivariate analysis system New York: Applied Biostatistics; 2000, Version 2.11X

22. Perrier $X$, Jacquemoud-Collet JP. DARwin software. 2006. http://darwin. cirad.fr. Accessed 10 Aug 2015

23. Pritchard JK, Stephens M, Donnelly P. Inference of population structure using multilocus genotype data. Genetics. 2000;155:945-59.

24. Evanno G, Regnaut S, Goudet J. Detecting the number of clusters of individuals using the software STRUCTURE: a simulation study. Mol Ecol. 2005; 14:2611-20.

25. Earl DA, vonHoldt BM. Structure Harvester: a website and program for visualizing STRUCTURE output and implementing the Evanno method. Conserv Genet Resour. 2012. doi:10.1007/s12686-011-9548-7.

26. Mateescu RG, Zhang Z, Tsai K, Phavaphutanon J, Burton Wursten NI, Lust G, Quaas R, Murphy K, Acland GM, Todhunter RJ. Analysis of allele fidelity, polymorphic information content, and density of microsatellites in a genome-wide screening for hip dysplasia in a crossbreed pedigree. J Hered. 2005. doi:10.1093/jhered/esi109.

27. Tessier C, David J, This P, Boursiquot JM, Charrier A. Optimization of the choice of molecular markers for varietal identification in Vitis vinifera $\mathrm{L}$. Theor Appl Genet. 1999;98:171-7.

28. Li YC, Korol AB, Fahima T, Beiles A, Nevo E. Microsatellites: genomic distribution, putative functions and mutational mechanisms: a review. Mol Ecol. 2002. doi:10.1046/j.1365-294X.2002.01643.x.

29. Morgante M, Hanafey M, Powell W. Microsatellites are preferentially associated with nonrepetitive DNA in plant genomes. Nat Genet. 2002. doi:10.1038/ng822.

30. Anderson JA, Churchill GA, Autrique JE, Tanksley SD, Sorrells ME. Optimizing parental selection for genetic linkage maps. Genome. 1993. doi:10.1139/g93-024.

31. Renvoize SA, Clayton WD, Kabuye CHS. Morphology, taxonomy and natural distribution of Brachiaria (Trin.) Griseb. In: Miles JW, Maass BL, Valle CB, editors. Brachiaria: Biology, agronomy, and improvement. EMBRAPA/ CIAT;1996. p. 1-15.

32. Jungmann $L$, Vigna $B B$, Boldrini $K R$, Sousa $A C$, do Valle $C B$, Resende $R M$, Pagliarini MS, Zucchi MI, de Souza AP. Genetic diversity and population structure analysis of the tropical pasture grass Brachiaria humidicola based on microsatellites, cytogenetics, morphological traits, and geographical origin. Genome. 2010. doi:10.1139/g10-055.

33. Valle CB, Savidan Y. Genetics, cytogenetics, and reproductive biology of Brachiaria. In: Miles JW, Maass BL, Valle CB, editors. Brachiaria: biology, agronomy, and improvement. EMBRAPA/CIAT; 1996. p. 147-63.

34. Valle CB, Miles JW. Breeding of apomictic species. In: Savidan Y, Carman JG, Dresselhaus T, editors. The flowering of apomixis: from mechanisms to genetic engineering. Mexico: CYMMYT/IRD; 2001. p. 137-52.

\section{Submit your next manuscript to BioMed Central and we will help you at every step:}

- We accept pre-submission inquiries

- Our selector tool helps you to find the most relevant journal

- We provide round the clock customer support

- Convenient online submission

- Thorough peer review

- Inclusion in PubMed and all major indexing services

- Maximum visibility for your research

Submit your manuscript at www.biomedcentral.com/submit
() Biomed Central 